\title{
REDUCED BRAIN SIZE OF VERY PRETERM INFANTS AT TERM EQUIVALENT AGE CAN BE ESTIMATED USING CEREBRAL ULTRASOUND MEASUREMENTS
}

\author{
A. Graca $^{1,2}$, K. Cardoso ${ }^{3}$

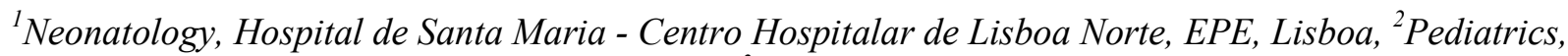 \\ Faculdade de Medicina - Universidade de Lisboa, ${ }^{3}$ Pediatrics, Hospital de Santa Maria - Centro Hospitalar \\ de Lisboa Norte, EPE, Lisbon, Portugal
}

Objective: To compare brain size between very preterm infants at term equivalent age (TEA) and term neonates using cerebral ultrasound (cUS) measurements and occipito-frontal head circumference (OFHC).

Methods: We assessed prospectively a cohort of 88 preterm infants $<32$ weeks gestation at TEA (mean postmenstrual age (PMA) 40.6 weeks) and 61 term controls (mean postnatal day 5.29, mean PMA 39.9 weeks). Infants with birth weight $<10^{\text {th }}$ centile or major scan abnormality were excluded. A single observer made cUS measurements of biparietal diameter, brain structures, ventricles, extracerebral spaces and also OFHC. A mathematical model was built to estimate from the cUS measurements and OFHC the axial area of head, CSF-filled spaces and brain tissue. Statistical comparison was performed using independent samples ttest or Mann-Whitney test when appropriate and corrected for PMA at scan.

Results: Preterms had a larger OFHC at TEA than controls $(\mathrm{p}=0.000)$, but total axial cranial area was similar $(p=0.705)$. However, the CSF-filled spaces were significantly larger in preterm $(p=0.000)$ than controls and estimated brain area in preterms was significantly smaller $(\mathrm{p}=0.000)$.

Conclusions: Our data shows that estimated brain area in an axial plane is smaller in preterms, supporting the view that extra-uterine preterm brain growth is compromised, even in the absence of overt lesions. Preterm children without major lesions but smaller brains may be more at risk of neurodevelopmental problems (Horsch 2005) and our data suggests that cUS measurements may be useful in their identification. Long-term follow-up is being undertaken in this cohort. 